\title{
Complicaciones y factores de riesgo en pacientes sometidos a colagiopancreatografía retrógrada endoscópica en una clínica de tercer nivel de Medellín desde el 2014 hasta el 2017
}

\author{
Complications and risk factors in patients undergoing endoscopic \\ retrograde cholangiopancreatography in a third level clinic in Medellín \\ from 2014 to 2017
}

Juan Sebastián Garcés ${ }^{1}$, Paula Andrea Mejía ${ }^{1}$, Javier Andrés Serrano ${ }^{1}$, Andrés Uribe ${ }^{2}$, Andrés Velásquez ${ }^{1}$, Juan Javier Agudelo ${ }^{3}$, Mauricio Moreno ${ }^{4}$, José Hugo Arias ${ }^{5}$

Médico y cirujano, Universidad del Valle; estudiante de especialización en Cirugía General, Universidad CES, Medellín, Colombia
Médico cirujano, Universidad de Caldas; estudiante de especialización en Cirugía General, Universidad CES, Medellín, Colombia
3 Médico cirujano, Universidad San Martín; especialista en Cirugía General, Universidad CES, Medellín, Colombia
4 Médico y cirujano, Universidad CES; especialista en Cirugía General, Universidad CES, Medellín, Colombia; subespecialista en
Cirugía Gastrointestinal y Endoscopia Digestiva, Universidad de Concepción de Chile, Concepción, Chile
Médico y cirujano, Universidad del Quindío; especialista en Anestesiología y Reanimación, Universidad Pontifica Javeriana; espe-
cialista en Epidemiología Clínica, Universidad El Bosque; estudiante de doctorado en Epidemiología, Universidad CES, Medellín,
Colombia

Tercer puesto, Concurso Nacional del Residente Quirúrgico. 45 Congreso, Semana Quirúrgica Nacional, agosto de 2019, Bogotá, D.C., Colombia

\section{Resumen}

Introducción. La colangiopancreatografía retrógrada endoscópica es la herramienta de elección para el manejo de una gran cantidad de enfermedades pancreáticas y biliares. Al ser un procedimiento invasivo, trae consigo riesgos que aumentan la morbimortalidad en los pacientes en quienes se practica. El objetivo de este estudio fue analizar las complicaciones más prevalentes relacionadas con el procedimiento, de los pacientes de una institución de tercer nivel de Medellín, entre los años 20I4 y 2017.

Métodos. Se presenta un estudio observacional, retrospectivo y analítico. Se analizaron las historias clínicas de los pacientes sometidos a colangiopancreatografía retrógrada endoscópica con diagnóstico de enfermedades biliares y pancreáticas. La información fue analizada usando estadística descriptiva e inferencial, mediante

Fecha de recibido: 15/06/2019 - Fecha aceptación: 21/08/2019

Correspondencia: Juan Sebastián Garcés, Carrera 38 N²6-343, torre 1, apartamento 211, Castellón de San Diego, Medellín, Colombia, 050016.

Correo electrónico: jsebas9@hotmail.com

Citar como: Garcés JS, Mejía PA, Serrano JA, Uribe A, Velásquez A, Agudelo JJ, et al. Complicaciones y factores de riesgo en pacientes sometidos a colagiopancreatografía retrógrada endoscópica en una clínica de tercer nivel de Medellín desde el 2014 hasta el 2017. Rev Colomb Cir. 2020;35:57-65. https://doi.org/10.30944/20117582.588.

Este es un artículo de acceso abierto bajo una Licencia Creative Commons - BY-NC-ND https://creativecommons.org/licenses/by-ncnd/4.0/deed.es 
análisis univariado y multivariado, y se calculó la razón de momios (odds ratio, OR) con un intervalo de confianza del $95 \%$; se tomó como significativo un valor de p inferior a o,25 en los análisis bivariados.

Resultados. En los I.546 pacientes sometidos a colangiopancreatografía retrógrada endoscópica, la edad promedio fue de 57,6 \pm I9.3 años y el 59,I \% eran mujeres. Las complicaciones identificadas fueron: pancreatitis en 57 pacientes $(3,7 \%)$, sangrado en $28(\mathrm{I}, 8 \%)$, perforación en $8(0,5 \%)$ y mortalidad no discriminada de 42 pacientes $(2,7 \%)$. Como factores de riesgo con significancia estadística, se encontraron la papilotomía y el contraste del conducto de Wirsung ( $\mathrm{OR}=3$ y $\mathrm{OR}=3,55$, respectivamente).

Discusión. Los resultados obtenidos se encuentran en concordancia con los de la literatura mundial, con tasas de complicaciones y mortalidad similares. Por otra parte, el perfil sociodemográfico de la población de estudio difiere de las cifras internacionales, con predominancia de la enfermedad estudiada en edades mayores y con mayor cantidad de comorbilidades.

Palabras clave: conducto colédoco; colangiopancreatografía retrógrada endoscópica; complicaciones; pancreatitis; perforación intestinal; hemorragia

\begin{abstract}
Introduction: Endoscopic retrograde cholangiopancreatography is the tool for the management of a large number of pancreatic and biliary diseases. Being an invasive procedure, it brings risks that increase morbidity and mortality in patients in whom it is practiced. The objective of this study was to analyze the most prevalent complications related to the procedure of the patients of a third level institution in Medellin between 2014 and 2017.

Methods: An observational, retrospective and analytical study is presented. The information was analyzed using descriptive and inferential statistics, by univariate and multivariate analysis, and the odds ratio (odds ratio, OR) was calculated with a $95 \%$ confidence interval; a value of $p$ less than 0.25 was taken as significant in the bivariate analyzes.

Results: In the I,546 patients who underwent endoscopic retrograde cholangiopancreatography, the average age was $57.6 \pm 19.3$ years and $59.1 \%$ were women. The complications identified were: pancreatitis in 57 patients (3.7\%), bleeding in 28 (I.8\%), perforation in 8 (0.5\%) and non-discriminated mortality of 42 patients (2.7\%). As risk factors with statistical significance, papillotomy and Wirsung duct contrast were found ( $\mathrm{OR}=3$ and $\mathrm{OR}=3.55$, respectively).

Discussion: The results obtained are consistent with those of the world literature, with similar complication and mortality rates. On the other hand, the sociodemographic profile of the study population differs from international figures, with a predominance of the disease studied in older ages and with a greater number of comorbidities.
\end{abstract}

Key words: common bile duct; cholangiopancreatography, endoscopic retrograde; complications; pancreatitis; intestinal perforation; hemorrhage.

\section{Introducción}

La colangiopancreatografía retrógrada endoscópica es una herramienta predominantemente terapéutica, de elección para una amplia variedad de enfermedades biliares y pancreáticas, tanto benignas como malignas. A pesar de su gran utilidad y versatilidad terapéutica, este procedimiento lleva consigo riesgos que, si bien ocurren con poca frecuencia, incrementan la morbilidad global de los pacientes sometidos al mismo, lo cual aumenta de manera ostensible los costos de la atención en salud. La prevalencia de las complicaciones globales reportadas en la literatura oscila entre el 5 y el Io \% de todas las colangiopancreatografías retrógradas endoscópicas, independientemente de la indicación para su práctica ${ }^{\mathrm{I}-3}$. 
En orden de prevalencia, las complicaciones más comúnmente asociadas al procedimiento son las pancreatitis posteriores a este procedimiento, el sangrado y la perforación de víscera hueca o de los conductos biliares o pancreáticos.

La pancreatitis asociada con la colangiopancreatografía retrógrada endoscópica tiene una incidencia global reportada entre el 3 y el Io $\%{ }^{4}$. Su presentación es variable y puede cursar dentro del espectro de las pancreatitis leves a graves, que conllevan un alto riesgo de mortalidad global de los pacientes. El sangrado se presenta en 0,2 a $2 \%$ de los pacientes intervenidos con este método ${ }^{4}$; generalmente, es clasificado como leve y en la mayoría de los casos es intraluminal, aunque puede presentarse como un hematoma hepático o intraabdominal. Por último, la perforación después de este tipo de endoscopia es la más infrecuente de las complicaciones, con una incidencia cercana al o,6 \% y una mortalidad tan alta como el $23 \%{ }^{4}$. Es difícil detectarla durante el acto operatorio, durante el cual se logra identificar la ruptura en menos de un tercio de los pacientes; se requiere una gran sospecha diagnóstica para iniciar su enfoque diagnóstico y terapéutico.

Se han estudiado diferentes factores de riesgo que favorecen la presentación de estas complicaciones, algunos relacionados con el operador o con el procedimiento y, otros, con el paciente. Aquellos que tienen una mayor relevancia en la práctica clínica son: el entrenamiento del endoscopista, la edad del paciente, la canulación difícil de la vía biliar, el corte antes o después de la esfinterotomía, la canulación e inyección de contraste en el conducto pancreático, los antecedentes de pancreatitis posterior a una colangiopancreatografía retrógrada endoscópica, las coagulopatías y las infecciones activas de la vía biliar ${ }^{4-8}$.

Debido al avance de la tecnología y a la posibilidad de mayor accesibilidad a este tipo de procedimientos, el número de colangiopancreatografías retrógradas endoscópicas practicadas se ha incrementado de manera exponencial. Por esta razón, es importante tener siempre presente que, a pesar de la baja incidencia de las complicaciones del procedimiento, estas pueden llegar a ser catastróficas para el paciente y su familia. A medida que este procedimiento se establece como la medida terapéutica estándar para un número creciente de entidades que comprometen la vía biliar, se incrementan las complicaciones asociadas a su realización.

Si bien los diferentes factores de riesgo y la prevalencia de los eventos descritos previamente ya son ampliamente conocidos en la literatura científica mundial, se hace necesaria una evaluación con datos locales, que permita establecer un punto de partida para el desarrollo de estrategias basadas en las características demográficas de la población de la región, para que se pueda generar un impacto favorable en el bienestar de los pacientes sometidos a uno o varios de estos procedimientos.

En este estudio, desarrollado en un centro de referencia de Medellín, mediante una evaluación retrospectiva de los pacientes sometidos al procedimiento entre 2014 y 2017 , se busca analizar la prevalencia de las complicaciones más frecuentes relacionadas con la colangiopancreatografía retrógrada endoscópica, la tipificación de las características poblacionales de la región y la asociación de los diferentes factores de riesgo descritos en la literatura con el desarrollo de estas complicaciones, evaluando de manera crítica nuestros resultados y comparándolos con los estándares descritos a nivel mundial.

\section{Métodos}

Se llevó a cabo un estudio observacional, analítico, retrospectivo y longitudinal, en el que se buscaba determinar las complicaciones de la colangiopancreatografía retrógrada endoscópica (pancreatitis, sangrado y ruptura de víscera hueca, de conductos biliares o pancreáticos) y evaluar la asociación de los factores de riesgo del paciente y del procedimiento para su presentación. Los datos se recolectaron de manera retrospectiva mediante la evaluación de las historias clínicas, las descripciones operatorias, las imágenes diagnósticas y los resultados de laboratorio clínico de los pacientes sometidos a este procedimiento entre enero de 2014 y diciembre de 2017 . 
Se incluyeron los pacientes con diagnóstico confirmado o presuntivo de enfermedad biliar y pancreática, a quienes se les practicó una colangiopancreatografía retrógrada endoscópica durante el periodo descrito y que tuvieran completos todos los datos solicitados en el instrumento de recolección. Se analizaron todas las historias clínicas dentro del registro de la clínica para este periodo, y se excluyeron únicamente los pacientes que no tuvieran la información detallada del procedimiento, su evolución y los antecedentes.

La información recolectada fue tabulada en Microsoft Excel ${ }^{\mathrm{TM}}$, versiones 2013 y 20I8; posteriormente, se analizó mediante estadística descriptiva e inferencial, previo proceso de depuración de la base de datos. En la etapa descriptiva, se hicieron análisis univariados de cada una de las variables de interés, así como análisis bivariados referentes a las complicaciones estudiadas y previamente descritas. Se presentan los resultados de frecuencia utilizando medidas de tendencia central mediante análisis univariados y multivariados, y se calculó la razón de momios (odds ratio, OR) con un intervalo de confianza del $95 \%$; se tomó como significativo un valor de $\mathrm{p}$ inferior a 0,05 .

Un especialista en epidemiología, ajeno a la recolección de datos, analizó la información con ayuda del programa SPSS ${ }^{\mathrm{TM}}$, versión $2 \mathrm{I}$.

\section{Resultados}

En el periodo comprendido entre el 2014 y hasta el 2017 , se practicaron I.654 procedimientos codificados como pancreatografía retrógrada endoscópica en la Clínica CES de Medellín. Tras excluir los registros duplicados o con datos incompletos y aquellos procedimientos en los que no se intervino la vía biliar o la pancreática, se obtuvieron I.546 registros que corresponden a todo el grupo estudiado en el presente trabajo.

Entre las características demográficas, se encontró un promedio de edad de 57,6 \pm 19,3 años, con predominio del sexo femenino, con 9 I4 pacientes (59,I \%) (tabla I). Al evaluar las comorbilidades, se encontró que la hipertensión arterial sistémica $(\mathrm{n}=604 ; 39, \mathrm{I} \%)$, la disli-
Tabla 1. Características demográficas de los pacientes incluidos en el estudio $(\mathrm{N}=1.546)$

\begin{tabular}{|c|c|}
\hline Características poblacionales & n (\%) \\
\hline Edad (años) & $\begin{array}{l}15 \text { a } 103 \\
\text { (M: } 57,58)\end{array}$ \\
\hline \multicolumn{2}{|l|}{ Sexo } \\
\hline Masculino & $632(40,9)$ \\
\hline Femenino & $914(59,1)$ \\
\hline \multicolumn{2}{|l|}{ Comorbilidades } \\
\hline Hipertensión arterial & $604(39,1)$ \\
\hline Diabetes mellitus & $215(13,9)$ \\
\hline Cáncer & $152(9,8)$ \\
\hline Inmunosupresión & $58(3,8)$ \\
\hline Antiagregación plaquetaria & $104(6,7)$ \\
\hline Anticoagulación & $54(3,5)$ \\
\hline Enfermedad renal crónica & $73(4,7)$ \\
\hline Dislipidemia & $285(18,4)$ \\
\hline \multicolumn{2}{|l|}{ Indicación de CPRE } \\
\hline Coledocolitiasis & $996(64,4)$ \\
\hline Colangitis & $176(11,4)$ \\
\hline Estenosis maligna & $183(11,8)$ \\
\hline Fístula biliar & $48(3,1)$ \\
\hline Fístula pancreática & $16(1)$ \\
\hline Otros & $127(8,2)$ \\
\hline \multicolumn{2}{|l|}{ Tipo de CPRE } \\
\hline Urgente & $633(40,9)$ \\
\hline Electiva & $913(59,1)$ \\
\hline \multicolumn{2}{|l|}{ Características de riesgo en CPRE } \\
\hline Canulación difícil & $238(15,4)$ \\
\hline Papilotomía & $962(62,2)$ \\
\hline Canulación del conducto de Wirsung & $170(11)$ \\
\hline Contraste del conducto de Wirsung & $73(4,7)$ \\
\hline \multicolumn{2}{|l|}{ Diagnóstico posterior a la CPRE } \\
\hline Patología benigna & $1.172(75,8)$ \\
\hline Obstrucción maligna & $183(11,8)$ \\
\hline Otros & $191(12,4)$ \\
\hline \multicolumn{2}{|l|}{ Éxito del procedimiento } \\
\hline CPRE exitosa & $1.384(89,5)$ \\
\hline CPRE fallida & $162(10,5)$ \\
\hline \multicolumn{2}{|l|}{ Resultados adversos } \\
\hline Pancreatitis posterior a la CPRE & $57(3,7)$ \\
\hline Sangrado posterior a la CPRE & $28(1,8)$ \\
\hline Perforación posterior a la CPRE & $8(0,5)$ \\
\hline Muerte & $42(2,7)$ \\
\hline
\end{tabular}

CPRE: colangiopancreatografía retrógrada endoscópica 
pidemia $(\mathrm{n}=285 ; \mathrm{I} 8,4 \%)$ y la diabetes mellitus $(n=215 ; 13,9 \%)$ fueron las más prevalentes en la población de estudio.

La principal indicación para la colangiopancreatografía retrógrada endoscópica fue la coledocolitiasis en 996 pacientes $(64,4 \%)$, seguida por la estenosis maligna en $\mathrm{I} 83$ (II,8\%), la colangitis en I76 (II,4\%), la fístula biliar en 48 (3,I\%), la fístula pancreática en I6 (I \%) y otras causas en I27 (8,2\%). De los procedimientos practicados, $633(40,3 \%)$ fueron urgentes y I62 (IO,5\%) se describieron como fallidos.
Se encontró una tasa de complicaciones del 6,or \% en la totalidad de los procedimientos practicados y una mortalidad del $2,7 \%$. Al hacer el análisis bivariado de las características preoperatorias de los pacientes para presentar pancreatitis, sangrado o perforación, se encontró asociación con una p estadísticamente significativa para hipertensión arterial sistémica $(\mathrm{p}=0,044)$; por otro lado, la mayoría de características, entre ellas la inmunosupresión $(\mathrm{p}=0, \mathrm{I} 8)$ y el cáncer $(\mathrm{p}=\mathrm{O}, \mathrm{I} 8)$, no fueron estadísticamente significativas (tabla 2). Al hacer el análisis multivariado

Tabla 2. Análisis bivariado para pancreatitis aguda posterior a la colangiopancreatografía retrógrada endoscópica

\begin{tabular}{|c|c|c|c|}
\hline \multirow[t]{2}{*}{ Características de la población } & \multicolumn{3}{|c|}{ Pancreatitis } \\
\hline & Sí & No & $\mathbf{p}$ \\
\hline Edad (Me con RIQ) & $53,4(36-65)$ & $57,7(44-73)$ & 0,073 \\
\hline Sexo femenino, n (\%) & $36(63,2)$ & $878(59)$ & 0,528 \\
\hline \multicolumn{4}{|l|}{ Comorbilidades, n (\%) } \\
\hline Hipertensión arterial & $15(26,3)$ & $589(39,6)$ & 0,044 \\
\hline Diabetes mellitus & $7(12,3)$ & $208(14)$ & 0,718 \\
\hline Cáncer & $6(10,5)$ & $146(9,8)$ & 0,858 \\
\hline Inmunosupresion & $4(7)$ & $54(3,6)$ & 0,186 \\
\hline Anticoagulacion & $2(1,8)$ & $102(3,6)$ & 0,719 \\
\hline Antiagregacion & $1(3,5)$ & $53(6,9)$ & 0,428 \\
\hline Enfermedad renal crónica & $3(5,3)$ & $70(4,7)$ & 0,749 \\
\hline Dislipidemia & $8(14)$ & $277(18,6)$ & 0,383 \\
\hline \multicolumn{4}{|l|}{ Indicación CPRE, n (\%) } \\
\hline Coledocolitiasis & $37(64,9)$ & $959(64,4)$ & \\
\hline Colangitis & $7(12,3)$ & $169(11,3)$ & \\
\hline Estenosis maligna & $6(10,5)$ & $177(11,9)$ & \\
\hline Fistula biliar & $3(5,3)$ & $45(3)$ & \\
\hline Fístula pancreática & $0(0)$ & $16(1,1)$ & \\
\hline Otros & $1(1,8)$ & $38(2,6)$ & \\
\hline \multicolumn{4}{|l|}{ Tipo de CPRE } \\
\hline Urgente & $27(47,8)$ & $606(40,7)$ & 0,315 \\
\hline \multicolumn{4}{|l|}{ Características del procedimiento } \\
\hline Canulación difícil & $13(22,8)$ & $225(15,1)$ & 0,114 \\
\hline Papilotomía & $48(54,2)$ & $914(61,4)$ & 0 \\
\hline Canulación del conducto de Wirsung & $16(28,1)$ & $154(10,3)$ & 0 \\
\hline Contraste del conducto de Wirsung & $11(19,3)$ & $62(4,2)$ & 0 \\
\hline \multicolumn{4}{|l|}{ Diagnóstico posterior a la CPRE } \\
\hline Coledocolitiasis & $39(68,4)$ & $1061(71,3)$ & 0,643 \\
\hline Obstrucción maligna & $10(17,5)$ & $197(13,2)$ & 0,348 \\
\hline Retiro de endoprótesis & $3(5,3)$ & $182(12,2)$ & 0,112 \\
\hline Otros & $9(15,8)$ & $248(16,7)$ & 0,861 \\
\hline CPRE fallida & $4(7)$ & $158(10,6)$ & 0,385 \\
\hline $\begin{array}{l}\text { Estancia posterior a la CPRE (mediana } \\
\text { con RIQ) días }\end{array}$ & $7(4-12)$ & $2(1-4)$ & 0 \\
\hline Muerte & $6(14,3)$ & $36(85,7)$ & 0,004 \\
\hline
\end{tabular}

CPRE: colangiopancreatografía retrógrada endoscópica 
con estas variables, no se encontró asociación del desarrollo de las complicaciones con las características preoperatorias o demográficas del paciente. $\mathrm{Al}$ evaluar las características técnicas, se encontró cierta asociación de complicaciones posteriores al procedimiento con la papilotomía, la canulación difícil, la canulación y el contraste del conducto de Wirsung.

Para el análisis multivariado, se tomaron nuevamente todas las variables que tenían una p estadísticamente significativa $(\mathrm{p}<0,05)$ y se obtuvieron los siguientes resultados: los factores de riesgo con significación estadística para desarrollar pancreatitis después de la colangiopancreatografía retrógrada endoscópica, fueron la papilotomía $\left(\mathrm{OR}=3 ; \mathrm{IC}_{95} \%\right.$ I,28-5,84) y el contraste del conducto de Wirsung $\left(\mathrm{OR}=3,55 ; \mathrm{IC}_{95 \%} \mathrm{I}, 28-9,9\right)$. En cuanto a los otros resultados, en el análisis bivariado para sangrado se encontró asociación solo con la papilotomía ( $\mathrm{p}=0,028)$. En los casos de perforación de víscera hueca o de los conductos biliares o pancreáticos, se encontró asociación con la canulación difícil ( $\mathrm{p}=\mathrm{0}, \mathrm{023})$.

$\mathrm{Al}$ someter dichas variables al análisis multivariado para determinar la real asociación con el sangrado o la perforación después de la colangiopancreatografía retrógrada endoscópica, no se encontró ningún factor de riesgo asociado. No obstante, es necesario aclarar que el porcentaje total de sangrado o de perforación fue muy bajo, lo que hace que tenga bajo poder para detectar diferencias o para establecer si existe una asociación o si no la hay.

\section{Discusión}

Los eventos adversos asociados con la colangiopancreatografía retrógrada endoscópica han sido evaluados en múltiples estudios, incluso, la American Society for Gastrointestinal Endoscopy (ASGE) elaboró una guía al respecto ${ }^{4}$. Se estima que en los Estados Unidos las complicaciones asociadas con dicho procedimiento alcanzan costos superiores a los USD\$ I50 millones.

La pancreatitis posterior a la colangiopancreatografía retrógrada endoscópica es la más común de las complicaciones, y su diagnóstico genera una amplia controversia, pues el $75 \%$ de los pacientes presenta elevación transitoria de las enzimas pancreáticas, inclusive en rangos diagnósticos de pancreatitis, independientemente de que se presenten o no los síntomas característicos para su diagnóstico.

Hasta el momento, se propone la clasificación de Cotton de 1992 y la revisión de Atlanta de 2012 (tabla 3) para su diagnóstico de la pancreatitis y

Tabla 3. Criterios diagnósticos y clasificación de la pancreatitis posterior a la colangiopancreatografía retrógrada endoscópica

\begin{tabular}{|c|c|c|c|}
\hline & Leve & Moderado & Severo \\
\hline Cotton, et al. & $\begin{array}{l}\text { A) Pancreatitis clínica } \\
\text { y } \\
\text { B) Amilasas, por lo menos, } 3 \text { veces } \\
\text { el valor normal, después de } 24 \\
\text { horas del procedimiento } \\
\text { y } \\
\text { C) Requerir nueva hospitalización } \\
\text { o prolongación de la misma } \\
\text { planeada por } 2 \text { a } 3 \text { días. }\end{array}$ & $\begin{array}{l}\text { Pancreatitis que requiera } \\
\text { hospitalización por } 4 \text { a } 10 \text { días }\end{array}$ & $\begin{array}{l}\text { A) Hospitalización por más de } \\
10 \text { días. } \\
\text { o } \\
\text { B) Desarrollo de pancreatitis } \\
\text { hemorrágica, flegmón, } \\
\text { pseudoquiste o infección } \\
\text { o } \\
\text { C) Necesidad de drenaje } \\
\text { percutáneo o cirugía }\end{array}$ \\
\hline $\begin{array}{l}\text { Clasificación de } \\
\text { Atlanta }\end{array}$ & $\begin{array}{l}\text { A) Dos de las siguientes: } \\
\text { Dolor consistente con pancreatitis } \\
\text { aguda } \\
\text { Amilasas o lipasas mayor de } 3 \\
\text { veces el valor normal } \\
\text { Hallazgos imagenológicos } \\
\text { característicos } \\
\text { y } \\
\text { B) Sin disfunción de órgano ni } \\
\text { otros eventos adversos }\end{array}$ & $\begin{array}{l}\text { A) Falla de órgano transitorio por } \\
\text { menos de } 48 \text { horas } \\
\text { o } \\
\text { B) Eventos adversos sistémicos } \\
\text { o locales sin falla persistente de } \\
\text { órganos }\end{array}$ & $\begin{array}{l}\text { A) Falla de órgano único o } \\
\text { múltiple mayor de } 48 \text { horas } \\
\text { o } \\
\text { B) Síndrome de respuesta } \\
\text { inflamatoria sistémica } \\
\text { persistente }\end{array}$ \\
\hline
\end{tabular}

Reproducida y traducida con permiso número 4732521146321 de: Chandrasekhara V, Khashab MA, Muthusamy VR, Acosta RD, Agrawal D, Bruining DH, et al. Adverse events associated with ERCP. Gastrointestinal Endoscopy. 2017;85:32-47. 
para determinar su gravedad. Con base en esta definición y estos criterios, la incidencia global de pancreatitis evidenciada en las revisiones sistemáticas oscila entre el 3 y el ro $\%{ }^{4}$. En algunos estudios con un número importante de pacientes $(n=13.296)$, la tasa de pancreatitis alcanza hasta el I4,7 \% en quienes tienen alto riesgo 9 . La incidencia de pancreatitis posterior al procedimiento en el presente estudio fue de $3,7 \%$, lo cual concuerda con lo informado a nivel mundial 4 .

En cuanto a los factores de riesgo, por ser descriptivo el estudio no permite determinar una causalidad directa, pero sí determinar algunas asociaciones; para esto, se incluyeron en los análisis aquellos factores descritos en la literatura: canulación difícil, papilotomía, contraste o canulación del conducto de Wirsung. En cuanto al primero, algunos autores lo definen como intentos repetitivos durante más de 5 a Io minutos; en este estudio, se utilizó solamente la descripción del endoscopista, independientemente del tiempo empleado en su manipulación. La canulación difícil está relacionada con el número de intentos de canulación, los cuales aumentan el riesgo de presentar pancreatitis. La incidencia de pancreatitis en estos casos es del $3 \%$ cuando hay menos de cinco intentos, de 9 $\%$ con 6 y 20 intentos y hasta de $15 \%$ con más de 20 intentos $(\mathrm{OR}=2,8)^{6}$.

La esfinterotomía o papilotomía previa mejora la tasa de canulación exitosa, incluso, en varios estudios se ha evidenciado una reducción del riesgo de pancreatitis con un riesgo relativo de $0,29^{4}$. En el presente estudio no se discriminó entre papilotomías antes o después del intento de canulación. En un estudio de I.786 casos, la OR fue de 3,I para la papilotomía y de 2,28 para la canulación del conducto de Wirsung ${ }^{\text {IO }}$.

El contraste del conducto de Wirsung se ha descrito también como un factor de riesgo para pancreatitis $(\mathrm{OR}=\mathrm{I}, 58)^{\mathrm{II}}$. En el análisis multivariado en el presente estudio, se encontró asociación de la papilotomía $\left(\mathrm{OR}=3 ; \mathrm{IC}_{95 \%}\right.$ I,28-5,84) y el contraste del conducto de Wirsung $\left(\mathrm{OR}=3,55 ; \mathrm{IC}_{95 \%} \mathrm{I}, 28-9,9\right)$, con el desarrollo de esta complicación.
Se presentó sangrado en 28 (I,8 \%) pacientes, un porcentaje muy parecido al reportado por otros autores ${ }^{12,13}$. En las diferentes series, el "precorte" y la papilotomía se describen como factores de riesgo para el sangrado posterior a la colangiopancreatografía retrógrada endoscópica. Al comparar esto con lo encontrado en el presente estudio, el análisis bivariado demostró una asociación entre el sangrado y comorbilidades, como la hipertensión arterial sistémica y la inmunosupresión, y en el aspecto técnico, con canulación difícil, papilotomía y canulación del Wirsung.

La incidencia de perforación gastrointestinal encontrada en este estudio, también coincide con la informada por otros autores ${ }^{13-17}$. En los pacientes con esta complicación, se encontró una edad media de 74 años, similar a lo descrita por Preetha, et al. ${ }^{17}$, al igual que predominio del sexo femenino y comorbilidades como hipertensión arterial sistémica, dislipidemia y cáncer, sin que puedan establecerse claramente como factores de riesgo.

En el presente estudio, la mayoría de los procedimientos fueron de tipo electivo por coledocolitiasis y se acompañaron de papilotomía; $\sin$ embargo, no se encontró asociación entre la perforación y la práctica de papilotomía. También en la mayoría de ellos, la canulación se describió como difícil; en el análisis bivariado, esto indicaría una diferencia estadísticamente significativa al compararlo con los pacientes sin perforación, un hallazgo que no se había reportado en artículos similares.

Se encontró una mayor estancia hospitalaria en casos de perforación del duodeno en comparación con la población total del estudio y con los pacientes con sangrado posterior a la colangiopancreatografía retrógrada endoscópica. Debido a la poca prevalencia de este tipo de perforación, es difícil caracterizar posibles factores de riesgo ${ }^{18}$. No obstante, se han descrito factores asociados, como la alteración anatómica quirúrgica, por ejemplo, reconstrucción de tipo Billroth II 4, 77-19, hallazgo ausente en los pacientes de la cohorte analizada. 
En la literatura científica mundial, se ha demostrado una fuerte asociación entre papilotomía, canulación difícil y anticoagulación, con el desarrollo de perforación y sangrado después de la colangiopancreatografía retrógrada endoscópica ${ }^{4-6}$. En el presente estudio, se presentaron muy pocas de estas complicaciones, lo que le otorga un bajo poder a la muestra para encontrar una asociación, en contraste con lo reportado en series más grandes.

La baja tasa de complicaciones posiblemente esté asociada a varios factores. En Medellín, la Clínica CES es un centro de referencia para procedimientos endoscópicos como la colangiopancreatografía retrógrada endoscópica, por lo cual el volumen de pacientes es constantemente alto; además, el personal que los realiza está entrenado y cumple con los requisitos técnicos para hacerlo.

En este estudio, hubo limitaciones como su carácter retrospectivo, el tratarse de pacientes de un único centro de atención y la falta de seguimiento a largo plazo, lo que podría interferir a la hora de sacar conclusiones definitivas. Consideramos que, a partir de este trabajo, se pueden proponer diferentes líneas de estudio que pudieran contribuir en el desarrollo de estrategias de manejo y prevención de las complicaciones descritas.

El presente estudio no permite determinar la incidencia de mortalidad, ya que no se discriminó la mortalidad global de la atribuida al procedimiento o a sus complicaciones. En términos de la mortalidad atribuida a la pancreatitis posterior a la colangiopancreatografía retrógrada endoscópica, en diferentes series se describen valores de $0,7 \%$.

\section{Cumplimiento de normas éticas}

Conflicto de intereses. Ninguno de los autores reportó conflictos de interés.

Consentimiento informado. Este estudio fue aprobado por el Comité de Ética de la Clínica CES y el de la Universidad CES. De acuerdo con la Resolución 008430 de 1993 del Ministerio de Salud de Colombia, se consideró que la investigación no tenía riesgo para los pacientes por ser una revisión retrospectiva de historias clínicas, por lo tanto, no se diligenció el consentimiento informado.

Financiación. La Universidad CES financió las asesorías epidemiológicas y la Clínica CES administró la base de datos.

\section{Referencias}

I. McNicoll CF, St Hill CR. Choledocholithiasis. In: StatPearls. Treasure Island (FL): StatPearls Publishing; 2019. Disponible en https://www.ncbi.nlm.nih.gov/ pubmed $/ 28722990$

2. Dadhwal US, Kumar V. Benign bile duct strictures. Med J Armed Forces India. 2012;68:299-303. https://doi. org/IO.IoI6/j.mjafi.2012.04.014

3. Pu LZCT, Singh R, Loong CK, de Moura EGH. Malignant biliary obstruction: Evidence for best practice. Gastroenter Res Pract. 20I6;2016:I-7. https://doi. org/IO.II55/2016/329680I

4. Chandrasekhara V, Khashab MA, Muthusamy VR, Acosta RD, Agrawal D, Bruining DH, et al. Adverse events associated with ERCP. Gastrointest Endosc. 20I7;85:32-47. https://doi.org/IO.IOI6/j. gie.2016.06.05I

5. Freeman ML. Complications of endoscopic retrograde cholangiopancreatography: Avoidance and management. Gastrointest Endosc Clin N Am. 2012;22:567-86. https://doi.org/IO.IOI6/j.giec.20I2.05.00I

6. Thaker AM, Mosko JD, Berzin TM. Post-endoscopic retrograde cholangiopancreatography pancreatitis. Gastroenterol Rep. 2015;3:32-40. https://doi.org/IO.IO93/ gastro/gouo83

7. Pannu DS, Draganov PV. Therapeutic endoscopic retrograde cholangiopancreatography and instrumentation. Gastrointest Endosc Clin N Am. 2012;22:4OI-I6. https://doi.org/IO.IoI6/j.giec.2012.05.003

8. Lin WC, Lin $\mathrm{HH}$, Hung CY, Shih SC, Chu CH. Clinical endoscopic management and outcome of post-endoscopic sphincterotomy bleeding. PLoS One. 2017;I2:eoI77449. https://doi.org/Io.I37I/journal. pone.0I77449

9. Li G, Wang F, Fang J, Zha H, Zhao Q. Risk factors for post-endoscopic retrograde cholangiopancreatography pancreatitis: Evidence from 1786 cases. Med Sci Monit. 20I8;24:8544-52. https://doi.org/IO.I2659/ MSM.9I33I4

Io. Kodali S, Mönkemüller K, Kim H, Ramesh J, Trevino J, Varadarajulu S, et al. ERCP-related perforations in the new millennium: A large tertiary referral center IO-year experience. United European Gastroenterol J. 20I5;3:25-30. https://doi.org/IO.II77/20506406I4560784 
II. Cotton PB, Garrow DA, Gallagher J, Romagnuolo J. Risk factors for complications after ERCP: A multivariate analysis of II,497 procedures over I2 years. Gastrointest Endosc. 2009;70:80-8. https://doi.org/IO.IoI6/j. gie.2008.10.039

I2. Ghoz HM, Abu Dayyeh BK. Hemorrhagic complications following endoscopic retrograde cholangiopancreatography. Techniques in Gastrointestinal Endoscopy. 20I4;I6:I75-82. https://doi.org/IO.IOI6/j. tgie.2014.07.002

I3. Imbeth P, Guzmán I, Julliao H, Salej J, Galván C. Complicaciones y factores de riesgo en pacientes llevados a colangiopancreatografía retrógrada endoscópica en el Hospital Militar Central en el periodo de enero de 20II a junio de 20I2. Revista Med. 20I4;22:20-7.

I4. Tavusbay C. The management of perforation after endoscopic retrograde cholangiopancreatography (ERCP). Ulus Travma Acil Cerrahi Derg. 20I6;22:44I-8.
I5. Masci E, Toti G, Mariani A, Curioni S, Lomazzi A, Dinelli $\mathrm{M}$, et al. Complications of diagnostic and therapeutic ERCP: A prospective multicenter study. Am J Gastroenterol. 200I;96:417-23.

I6. Silviera ML, Seamon MJ, Porshinsky B, Prosciak MP, Doraiswamy VA, Wang CF, et al. Complications related to endoscopic retrograde cholangiopancreatography: A comprehensive clinical review. J Gastrointestin Liver Dis. 2009;18:73-82.

I7. Preetha M, Chung YF, Chan WH, Ong HS, Chow PK, Wong WK, et al. Surgical management of endoscopic retrograde cholangiopancreatography-related perforations. ANZ J Surg. 2003;73:IOII-4.

18. Guda NM, Reddy DN, Kumar A. Complications of ERCP. Indian J Gastroenterol. 2014;33:I-9. https://doi. org/IO.IOO7/sI2664-0I3-0383-5

19. Freeman ML. Adverse outcomes of ERCP. Gastrointest Endosc. 2002;56:S273-82. 